\title{
CLUSTERS OF GALAXIES AND THE HOT INTRACLUSTER
} MEDIUM

\author{
C. Jones, W. Forman and L. David \\ Harvard/Smithsonian Center for Astrophysics, 60 Garden St., \\ Cambridge, MA
}

\begin{abstract}
The luminous material in clusters of galaxies falls primarily into two forms - the visible galaxies and the $\mathrm{X}$-ray emitting intracluster medium. The hot intracluster medium is the major observed baryonic component of clusters with a mass equal to or greater than that of the stellar matter. In this paper we discuss changes in the efficiency of galaxy formation for different clusters and the origin of the intracluster medium.
\end{abstract}

\section{THE ORIGIN OF THE INTRACLUSTER MEDIUM AND THE EFFICIENCY OF GALAXY FORMATION}

The ICM is a major component of the cluster being equal or greater in mass than the stellar matter. It is of particular importance to determine the origin of such a large fraction of the known baryonic mass of the cluster. Equally fundamental and related problems to address are the effects of the ICM on the morphology of galaxies and the efficiency of galaxy formation. To begin to address the questions of the origin of the ICM and the efficiency of galaxy formation in different environments, it is useful to compare the ratio of gas mass to stellar mass in groups of galaxies and rich clusters. It is well known that the mass-to-light ratio increases with the size of the system. However, from poor to rich clusters the fraction of X-ray emitting gas to virial mass remains relatively constant $(\sim 10 \%$ within the central five core radii) (e.g. Abramopolilos and $\mathrm{Ku}, 1983$ ). Therefore, the ratio of gas mass to stellar mass should increase from the poor to rich clusters. As shown in Figure 1, in groups of galaxies the gas mass is approximately equal to the stellar mass, while in very rich 
clusters, the gas mass exceeds the stellar mass by as much as a factor of six (David et al. 1988).

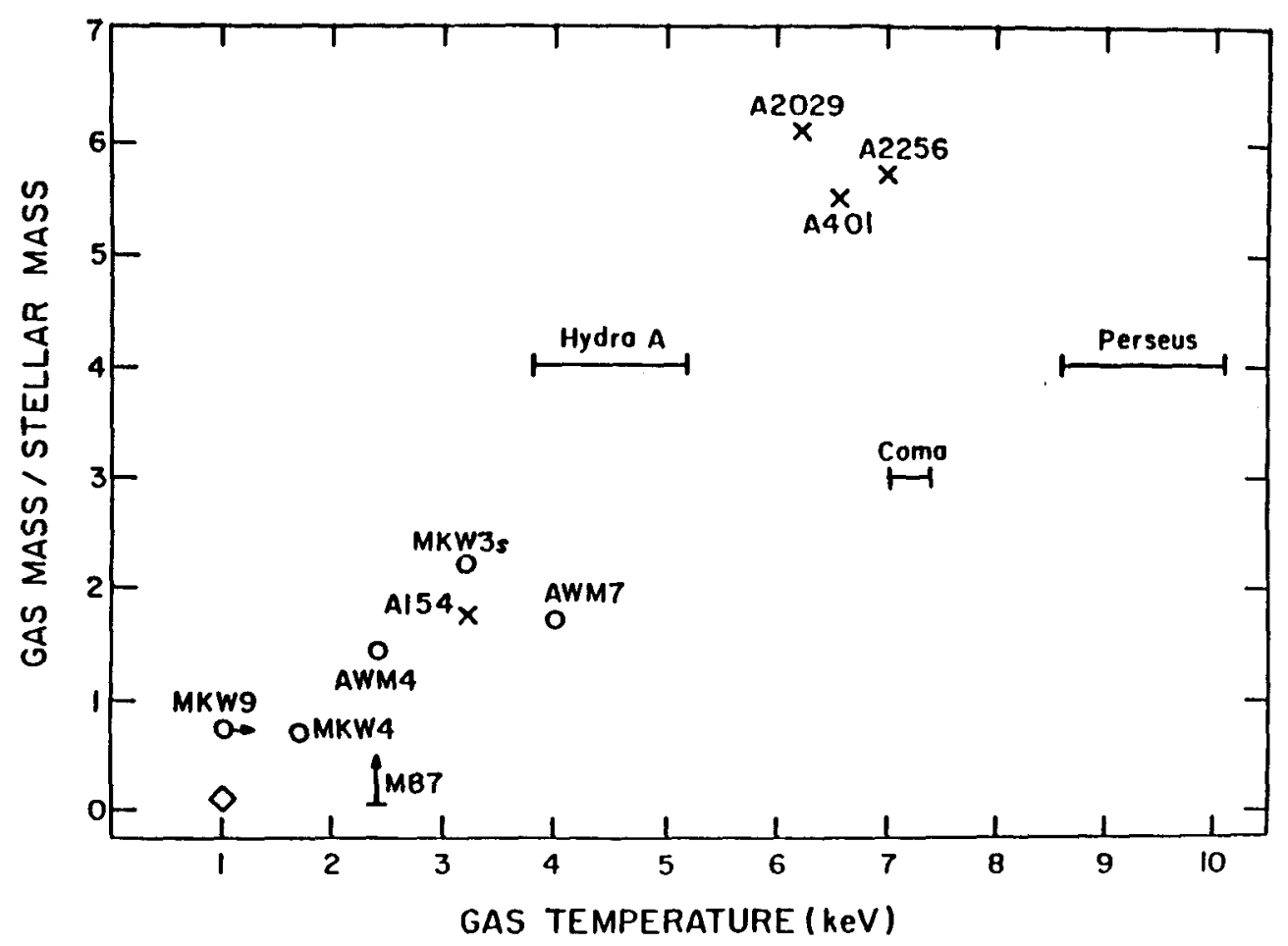

Fig. 1. The ratio of the gas mass as measured by the X-ray observations to the stellar mass is plotted against the temperature of the gas. The gas and stellar masses are evaluated within five core radii. The temperature of the gas reflects the gravitational potential of the system. The increasing ratio of gas mass to stellar mass with increasing gas temperature suggests a decreasing efficiency of galaxy formation (conversion of gas to luminous stellar matter) between groups and rich clusters.

The discovery of heavy elements in the ICM (Mitchell et al 1976, Serlemitsos et al. 1977) revealed an entirely new aspect to the study of the ICM - one crucial in 
determining its origin. Since heavy elements can be produced only through thermonuclear reactions in stars or by supernovae, the discovery that the intracluster medium was enriched in heavy elements required that material processed through stars be ejected into the ICM. The near solar abundance of the ICM measured by early X-ray experiments led to the suggestion that a large fraction of the material in the ICM was ejected from galaxies (e.g. DeYoung, 1978).

While the enriched material must come from the galaxies (in the absence of a Population III component), more recent studies have suggested that the bulk of the ICM of a rich cluster could not have originated within the galaxies because its mass is several times larger than the mass of the galactic stellar component. Thus, the bulk of the ICM in rich clusters must be "left over" from the formation of the galaxies. In particular, numerical modelling of the hot gaseous coronae around elliptical galaxies shows that over a Hubble time these galaxies can contribute only a fraction of their stellar mass to the IGM (David, Forman and Jones 1989).

While such an analysis constrains the contribution from present epoch galaxies to the ICM, if we assume that the groups and clusters are "closed" systems, we can use the ratios of the stellar mass to gas mass in groups and clusters to limit both the mass loss from present epoch galaxies to the ICM as well as any contribution from early, population III stars. Specifically, so long as the IMF's and the population III component of groups and clusters are similar and no gas is lost or gained by the system, then we would expect the stellar contribution to the ICM per unit stellar mass to be the same in all groups and clusters. The approximate equality of gas mass and stellar mass in the low X-ray luminosity Morgan groups (MKW4, MKW9, and AWM4) limits the contribution to the ICM by all stars to no more than the present stellar mass. Thus, in the richest clusters where the gas mass is three to six times the stellar mass, only a small fraction of the ICM could have been produced in stars. In the rich clusters, most of the gas in the intracluster medium must be primordial.

The ratio of the gas mass to the stellar mass, $M_{\text {gas }} / M_{\text {stellar }}$, shown in Figure 1, can be related to the efficiency of star formation. We assume a scenario in which the luminous matter (stars and the ICM) form the bulk of the baryonic material and the remainder of the virial mass is in the form of hot or cold dark matter. Then the efficiency of galaxy formation, the conversion of baryons from gas to stars in galaxies, can be written as

$$
\epsilon=M_{\text {stellar }} / M_{\text {lum }}
$$

where $M_{l u m}=M_{\text {stellar }}+M_{\text {gas }}$, or equivalently as

$$
\epsilon=\left(1+M_{\text {gas }} / M_{\text {stellar }}\right)^{-1}
$$


(assuming the expelled gas from galaxies is small and can be neglected). Thus by measuring $M_{\text {gas }} / M_{\text {stellar }}$ we can study the efficiency of star formation in systems ranging from groups to rich clusters. Our analysis shows that the star (and galaxy) formation efficiency ranges from $50 \%$ for groups to as little as $\approx 15 \%$ for rich clusters. If all the ICM in groups is instead gas ejected from galaxies, and we use this injection rate for all clusters, then while the galaxy formation efficiency would be $100 \%$ for groups, a lower efficiency is still found for rich clusters (as low as $\approx 17 \%$ for $\mathrm{M}_{\text {gas }} / \mathrm{M}_{\text {stellar }}=6$ to as high as $\approx 50 \%$ for $\mathrm{M}_{\text {gas }} / \mathrm{M}_{\text {stellar }}=3$ ). Although the amount of luminous material (gas+stars) remains relatively constant for all clusters (Blumenthal et al. 1984), the efficiency of galaxy formation decreases as one moves to richer systems. In other words, although the richest systems obviously produced more galaxies, their efficiency of galaxy formation was lower.

In such a "closed" cluster system, as long as the galaxies in different clusters have similar initial mass functions so that the amount of enriched material is directly related to the stellar mass, then the decrease in efficiency of galaxy formation between groups and rich clusters implies that the intracluster medium in poor clusters should show a greater abundance of heavy elements than that in rich clusters. Since the conclusion concerning different enrichments of the ICM hinges on clusters and groups having similar IMF's, it is important to note that Morgan, Kayser and White (1975) found the galaxy populations of many of the $\mathrm{cD}$ dominated groups to be similar to those of rich Abell clusters of Bautz-Morgan Type I. There is no evidence that the galaxies in rich clusters are significantly different from the galaxies in these dense groups.

Since it is likely that the galaxies in rich and poor clusters formed with similar IMF's, the iron (and other heavy elements) produced during the evolution of the component stars would yield a mass of iron directly proportional to the present epoch stellar light of the galaxies:

$$
M_{F e}=\eta L_{\text {galaxies }}
$$

where $\eta$ is determined by the IMF. Let us assume that the fraction of material ejected from the galaxies into the ICM is a constant fraction of the material liberated by the constituent stars. This is supported observationally by the similar colors of Morgan group galaxies and cluster galaxies implying comparable metal abundances. Further support comes from the similarity in the effectiveness of gas removal mechanisms. Internal mechanisms (e.g. supernova driven galactic winds) would be indifferent to the environment and ram pressure stripping (proportional to $\rho v^{2}$ ) of the gas from galaxies is comparable in groups and clusters (the gas temperature, a measure of $v^{2}$, increases by a factor of 3-4 from groups to rich clusters and the central gas densities are perhaps slightly higher in groups and hence partially 
offsetting). Thus, in the simplest scenario we can predict a trend of iron abundance with the depth of the potential of the system - increasing potentials, correlated with lower galaxy formation efficiencies, imply decreasing iron abundances.

The present measurements of iron abundances are too inaccurate to verify the above model or test possibilities for the origin of the ICM. Mushotzky (1984) and Henriksen (1985) summarize present results. For rich clusters, Henriksen (1985) reports a possible correlation of decreasing iron abundance with increasing gas temperature, as predicted, but the data are not sufficiently precise to yield quantitative results. Those observations were for only quite luminous clusters $\left(L_{x}>2 \times 10^{44}\right.$ ergs $\sec ^{-1}$ ) while in general we expect the abundances to be highest in the low luminosity clusters. Hughes et al. (1988) have measured a precise iron abundance of $22 \%$ of the solar value for the rich Coma cluster. To adequately test for differences in abundances, it is particularly important to obtain comparable measurements for low temperature (high galaxy formation efficiency) systems. By determining accurate values of the heavy-element abundances of the ICM in both poor and rich clusters, one could better investigate the properties of the IMF (e.g. exponent), the efficiency of galaxy formation, and the origin and enrichment of the ICM. A precise determination of the heavy element abundance of the intracluster medium for a sample of clusters ranging from groups to rich clusters has implications for the amount of material in the ICM that must be primordial. In particular, determining a high solar abundance for the ICM in Morgan groups, as suggested by the arguments above, would confirm that the origin of most of the hot gas in rich clusters must be primordial.

The changing ratio of gas mass to stellar mass also will affect the energy (or temperature) of the ICM. By measuring the surface brightness profiles and independently by measuring the ratio of the velocity dispersion to the gas temperature, one can estimate the energy per unit mass of the galaxies compared to that of the gas. From the surface brightness profiles, this value for rich clusters is generally $\sim 2 / 3$. The values calculated from the measured velocity dispersions and gas temperatures have a wider range (but see Flanagan et al. (1989) who suggest a resolution for the Perseus discrepancy). By comparison to rich clusters, the surface brightness profiles for hot gas around single dominant cluster galaxies such as M87 and the cD groups such as AWM7 yield a value $\sim 1 / 2$ (Fabricant and Gorenstein 1983, Jones and Forman 1989). This implies that the groups and individual central galaxies have more energy per unit mass in gas compared to the constituent galaxies than do rich clusters. For the groups and poor clusters where the stellar mass is comparable to the gas mass, there may be significant heating of the ICM by the ejected material which may account for the observed difference between the groups and the clusters. 


\section{Acknowledgements}

We thank Karen Modestino and Donna Wyatt for their excellent preparation of this manuscript. This work was supported through NASA Contract NAS8-30751.

\section{REFERENCES}

Abramopoulos, F. and Ku, W. 1983, Ap.J., 271, 446.

Blumenthal, G.R., Faber, S.M., Primack, J.R., and Rees, M.J. 1984, Nature, 311, 517.

David, L., Forman, W., and Jones, C. 1989, in preparation.

DeYoung, D.S. 1978, Ap.J., 223, 47.

Fabricant, D. and Gorenstein, P. 1983, Ap.J., 267, 535.

Flanagan, J., Hughes, J., Arnaud, K., Forman, W., and Jones, C. 1989, in preparation.

Henriksen, M. 1985, Ph.D. Thesis (University of Maryland).

Hughes, J.P., Yamashita, K., Okumura, Y., Tsunemi, H., and Matsuoka, M. 1988, Ap.J., 327, 615.

Jones, C. and Forman, W. 1989, in preparation.

Morgan, W.W., Kayser, S., and White, R.A. 1975, Ap.J., 199, 545.

Mushotzky, R. 1984, Phys. Sci., T7, 157.

Serlemitsos, P.J., Smith, B.W., Boldt, E.A., Holt, S.S., and Swank, J.H. 1977, Ap.J., 211, L63. 